\title{
Immediate Effect of Self Stretching and Foam Roller Release on Tensor Fascia Lata Tightness: A Randomized Controlled Trial
}

\section{Khushali Choksi ${ }^{1 *}$, Dhvani Champaneri ${ }^{2}$ and Arti Patel ${ }^{2}$}

${ }^{1}$ Assistant Professor, S.S Agrawal Institute of Physiotherapy and Medical Care

Education, Navsari, India

${ }^{2}$ Intern of Bachelor of Physiotherapy, S.S Agrawal Institute of Physiotherapy and

Medical Care Education, Navsari, India

*Corresponding Author: Khushali Choksi, Assistant Professor, S.S Agrawal Institute

of Physiotherapy and Medical Care Education, Navsari, India.
Received: September 11, 2020

Published: September 25, 2020

(C) All rights are reserved by Khushali

Choksi., et al.

\begin{abstract}
Background of the Study: Tensor fascia lata muscle is commonly seen to be tight in normal healthy subjects and leads to Myofascial restriction. It causes range of motion restriction of hip and knee joint, pain at lumbopelvic area and knee pain.

Aims of the Study: The aim of the study was to evaluate the immediate effect of self stretching and foam roller release in subjects with TFL tightness.

Study Design: Randomized Controlled Trial.

Methodology: 90 subjects with TFL tightness were randomly allocated using chits for the study in three different groups with 30 subjects in each group. The outcome measure taken was OBER TEST; hip adduction range of motion. Pre and post treatment outcome measure was taken by Therapist 1 . The instruction for intervention was given by therapist 2 for Group A (self stretching) and was performed for three repetitions with 30 seconds hold and Group B (foam rolling) and was performed for two minutes. Group C was control group who did not receive any kind of intervention.

Result: There was statistical significant difference within foam roller and self stretching group. Using Post hoc analysis, there was significant difference between foam roller-control group and self stretching-control group (p value 0.000). There was no significant difference between foam roller and self stretching group ( $\mathrm{p}$ value 0.422 ).

Conclusion: There was statistical significant difference within self stretching and foam roller release group but when all three groups were compared simultaneously, self stretching and foam roller release group were equally effective in reducing the tightness of tensor fascia lata.
\end{abstract}

Keywords: TFL Tightness; Self Myofascial Release; Self Stretching; Ober Test; Foam Roller Release

\section{Abbreviations}

ROM: Range of Motion; ASIS: Anterior Superior Iliac Spine; TFL: Tensor Fascia Lata; ITB: Iliotibial Band; SMR: Self Myofascial Release; FR: Foam Roller Release; SS: Self Stretching; CC: Control Group

\section{Introduction}

Appropriate increase in ROM associated with short and long term flexibility training can positively affect the musculoskeletal health $[1,2]$. Flexibility may be hindered for a number of reasons, one of which is fascial restriction. Fascia becomes restricted because of injury, disease, inactivity, or inflammation. This restriction 
can decrease flexibility, strength, endurance, motor co-ordination and leads to high amount of physical pain [2].

Tensor fascia lata muscle is seen to be tight in the age group of 18 - 25 years and most commonly affects male population [3]. The TFL helps stabilize the knee when it is extended and stabilizes the trunk on the thigh. Because the ITB crosses two joints, disturbances in the kinematic chain in the lower extremity can lead to abnormal pelvic biomechanics [4]. As the involved tissues respond to extreme demand or stress, the ITB, gluteus maximus, gluteus medius and TFL tighten [5]. When TFL is tight, it limits hip adduction and lesser extent of hip extension, hip lateral rotation and hip flexion. Shortening of this muscle contributes to low back pain, Iliotibial band syndrome and patellofemoral pain due to lateral tracking of patella [6].

Iliotibial band syndrome is the most common cause of lateral knee pain in athletes. Patient with IT Band syndrome complains of pain along the lateral aspect of the knee, specifically at the lateral femoral condyle [7]. It is often assumed that IT Band syndrome results from abnormal increase in the compression forces between ITB and lateral epicondyle causes irritation and inflammation beneath the ITB [8]. Thus, it is important that tightness and flexibility of the TFL are kept normal to avoid ITB hardness.

For quantitative measurement of TFL tightness "OBER TEST" is performed with Goniometer measurement in degrees [3]. Modified Ober test creates less stress to the medial knee joint, less tension on the patella, less potential interference by a tight rectus femoris muscle, and a more functional test position. Based on review of the literature, the OBER TEST and Modified OBER TEST appear to be used with equal frequency; neither of the test has been shown to be more popular, more accurate and easier to perform than the others [9]. There are various therapeutic interventions like osteopathic soft-tissue manipulation, structural integration; various form of massage, muscle energy techniques, neurodynamics, stretching, myofascial release and Graston to improve mobility of the soft tissue and consequently increase ROM and flexibility [10].

Stretching exercises are commonly prescribed during warm-up and cool down protocols, strength and conditioning training programs, and rehabilitation programs. It is applied for the purpose of injury prevention, increasing joint range of motions and increasing muscle extensibility [11]. Static stretching is presented as a safer and more effective method because it does not exceed the normal range of motion of joints. It does not require a high level of fitness and causes less muscle pain [12].

Self Myofascial release (SMR) therapy is a manual-therapy technique developed by Barnes [1]. Common self-myofascial release tools include the foam roll and various types of roller massages. Foam Roller is a common tool used for self myofascial release. Foam Roller release is used to treat myofascial restrictions and restore muscles, tendons, ligaments, fascia, and/or soft-tissue extensibility [13]. These tools utilize the same mechanism of treatment as traditional myofascial release, but the pressure is applied by the subject externally or using body weight [14]. Foam rolling may be used as a warm-up without negatively effecting performance and may enhance joint mobility at the shoulder [15], lumbopelvic $[16,17]$, hip [18], knee [14,19-22] and ankle [23]. Duration of foam roller application has varied as well it is recommended that myofascial release occur for $60-90$ seconds to 5 minutes or until the release is felt [24]. There are various studies on effectiveness of Self Myofascial release using foam roller with and without joint range of motion of ankle and knee as well as effect of self myofascial release using foam roller on calf and quadriceps muscle [18,23]. There is one study which is done on effectiveness of stretching techniques in tensor fascia lata in patients with back pain [25]. There is study which is done to check the effect of foam rolling and static stretching on passive hip flexion range of motion which suggested the use of foam roller in combination of static stretch [18]. But there are no retrievable literatures available checking the effectiveness of foam roller and self stretching on tensor fascia lata muscle. In the present study the control group was also included which can demonstrate the need of intervention.

\section{Aim of the Study}

The aim of the study was to evaluate the immediate effect of self stretching and foam roller release in subjects with TFL tightness.

\section{Objective of the Study}

- Primary objective: To find out pre and post interventional effect of self stretching and foam roller release in subjects with TFL tightness.

- Secondary objective: To compare the effect of self stretching, foam roller release and control group following immediate treatment. 


\section{Significance of study}

The significance of the study was to check the effectiveness of self stretching and foam roller release in subjects with TFL tightness. If the results are found significant for any of the technique it can be used as an effective treatment for rehabilitation of subjects with TFL tightness.

\section{Materials and Methods}

Study design: Randomized controlled trial.

Sample size: 90 samples.

Sampling method: Purposive sampling.

Study duration: 6 months.

\section{Inclusion criteria}

- $\quad$ Age (18 - 25) years.

- $\quad$ Both males and females.

- $\quad$ Subject with tensor fascia lata tightness with positive Ober's (>10 degree of hip adduction) test.

- Subject with right or left lower limb tightness.

- $\quad$ Subject's willingness to participate.

\section{Exclusion criteria}

- $\quad$ Recent injury.

- $\quad$ Any fixed deformity or contracture.

- Any disability present.

- Recent surgery.

- History of Low back pain and knee pain.

\section{Material}

- Universal Goniometer.

- $\quad$ Commercial Foam Roller made up of high density Ethylenevinyl acetate; diameter of $15 \mathrm{~cm}$ and 36 inches length.

- Yoga mat

- $\quad$ Stop watch

- Plinth

- $\quad$ Recording sheet (Figure 1,2)

\section{Outcome measures}

Ober test: Reliability of Goniometer to measure tightness of TFL muscle by OBER test is established [26].

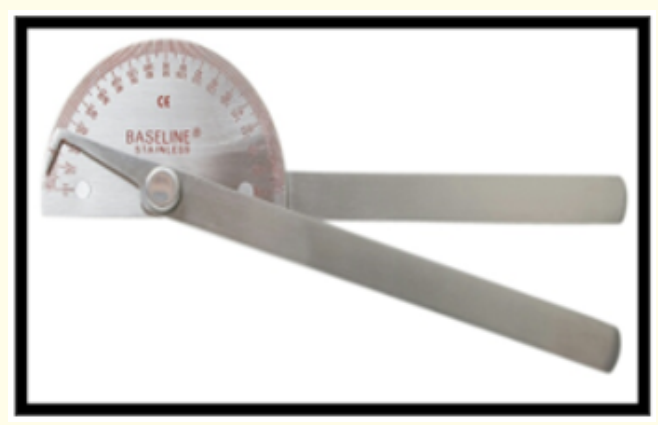

Figure 1: Universal goniometer.

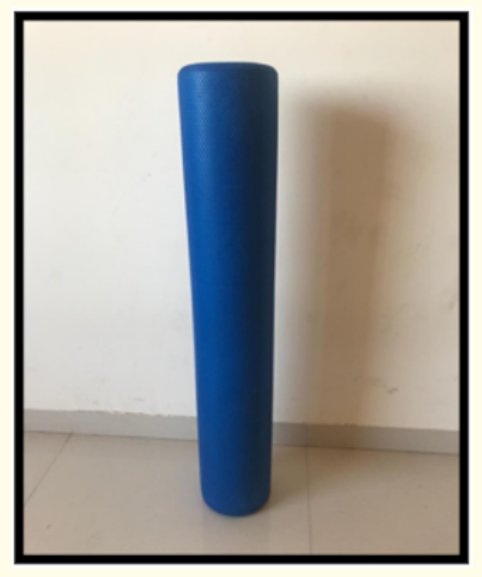

Figure 2: Foam roller.

Starting position: With the individual in side lying position near the edge of the examining table and the hip being tested in the uppermost position, the therapist stood directly behind the subject. Initially extending the uppermost knee and placing the hip in 0 degree of flexion, extension, abduction, adduction and rotation, the subjects were asked to flex the bottom hip and knee to stabilize the trunk, flatten the lumbar curve and keep the pelvis in slight posterior tilt.

Stabilization: The therapist 1 placed one hand on the iliac crest to stabilize the pelvis, firm pressure prevents the pelvis from laterally tilting during testing motion. The subjects were asked to flex the bottom hip and knee which also helped to stabilize the trunk and pelvis.

Testing motion: The therapist supported the leg being tested by holding the medial aspect of the knee and lower leg. The subject were asked to flex the hip and knee to 90 degree and keeping the 
knee flex, moving the hip into abduction and extension to position the TFL over greater trochanter of femur. The length of TFL was tested by lowering the leg into hip adduction and bringing it down towards examining table. The pelvis was not allowed to tilt laterally or hip to flex because these motion slacken the muscle. The knee was flexed to control medial rotation of hip and to maintain the stretch of muscle. The test was considered negative if the thigh drops to slightly below horizontal (10 degree of hip adduction), and the TFL and iliotibial band are of normal length. The test was considered positive if the thigh remains above horizontal in hip abduction and TFL and iliotibial band was tight (Figure 4).

Goniometer alignment: Central fulcrum of Goniometer was placed over ASIS of extremity being measured. The proximal arm was aligned with an imaginary line extending from one ASIS to other ASIS. The distal arm was aligned with anterior midline of femur using midline of patella for reference (Figure 3). The above procedure was adopted from Norkin [3].

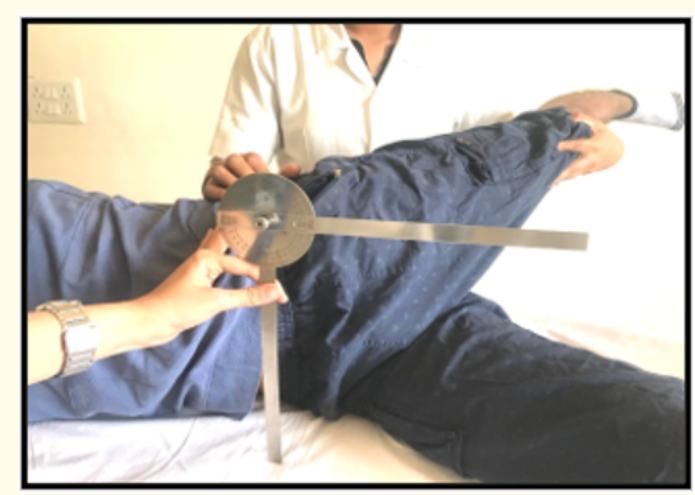

Figure 3: Alignment of goniometer.

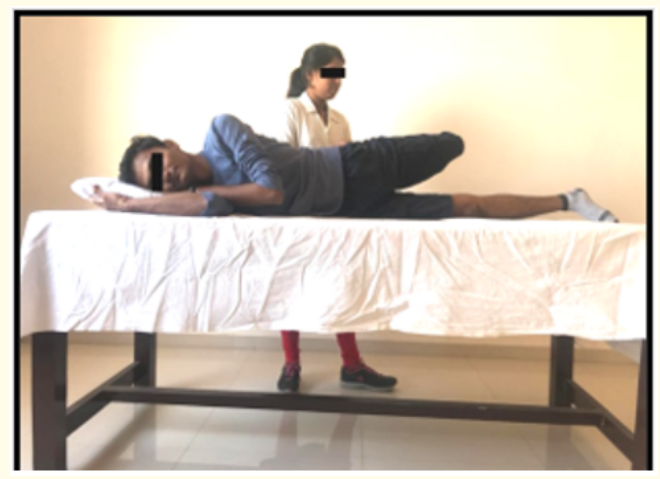

Figure 4: Positive Ober test.

\section{Details of intervention}

Self-stretching: The subjects were instructed to stand with the side to be stretch towards wall and hand on that side placed on the wall. The subject were asked to extend, adduct and externally rotate the extremity to be stretched and cross it behind the other extremity with both feet on the floor and shift his or her pelvis towards the wall and allow the normal knee to bend slight. There is a slight side bending of the trunk away from the side being stretch (Figure 5).

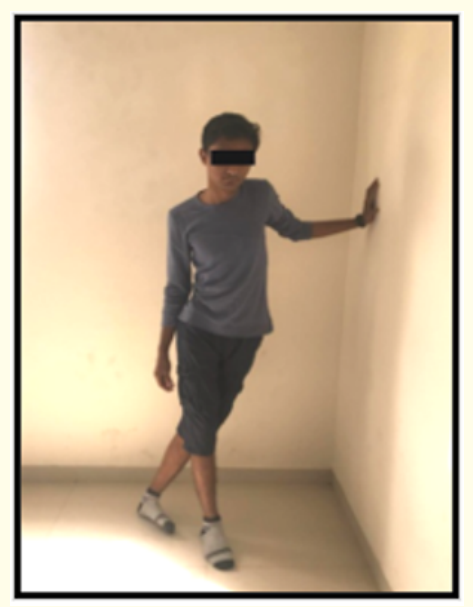

Figure 5: Self stretching.

Foam roller release: The subjects were asked to lie down in side lying position with the involved thigh on a foam roller positioned perpendicular to the femur. The subjects were asked to maintain the hip of the involved side in extension, flex the top hip and knee and plant the foot on the floor. They were asked to prop on the forearm or hands and lift the trunk and adduct the hip of the involved leg. Then subjects were asked to roll the lateral thigh using their body weight proximally and distally on the foam roller along the IT band (Figure 6). Both the intervention technique was adopted from Kisner [27].

\section{Procedure}

In this study total 90 subjects (male $=20$ and females $=70$ ) were included. The subjects were recruited from S.S. Agrawal group of colleges between the age group of 18 - 25 years with TFL tightness and were screened by therapist 1 and who fulfilled the inclusion and exclusion criteria were selected for study. The subjects who were screened were randomly allocated in 1 of the 3 treatment groups by selecting a number 1,2,3 from a container, where Group 
A- Self Stretching group, Group B-foam roller release group and Group C- Control group. All the three groups A, B, C had 30 samples each. Informed consent form was obtained from all subjects. All the subjects were explained about the procedure in detail. All subjects were tested by therapist 1 and were blinded from the results and subjects included in the study. They were asked to refrain from any strenuous exercise for at least three hours prior to testing as this may affects flexibility.

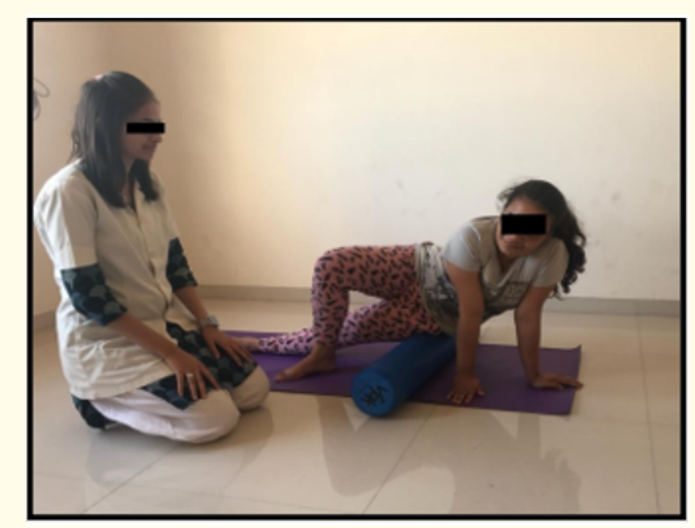

Figure 6: Foam roller release.

All subjects underwent one session of testing that included pre test measures, followed by intervention, then immediate post test measure. Then the testing for measuring tightness of TFL muscle was performed by Ober test with the protocol as described in outcome measure. Intervention was divided in 3 groups where therapist 2 had given self stretching instruction to group A and foam rolling instruction to group B. Non intervention Group was considered as control Group C. Subjects who were in group B were allowed to familiarize themselves with the foam roller. It consisted of giving subjects specific instructions on how to perform foam rolling and demonstration by the second therapist to ensure the correct technique. They were not allowed to practice on foam roller as this may influence the result.

Group A - Self Stretching: The subjects who entered in group A were given self stretching of TFL muscle instructed by therapist 2 for 3 repetitions of 30 seconds hold time. The outcome measure was taken by therapist 1 before and after intervention. Group B - Foam Roller Release: The subjects who entered in group B were given foam rolling instructions on TFL muscle by therapist 2 for 2 minutes. The outcome measure was taken by therapist 1 before and after the intervention. Group C - Control: The subjects who entered in group $\mathrm{C}$ were not given any of intervention. The outcome measure was taken by therapist 1 before and after 2 minutes.

\section{Results}

Data analysis was done using the SPSS software (version 20.0). Descriptive analysis was used to calculate mean and standard deviation. Normality of data was checked using the method of Kolmogorov-Smirnov and Shapiro-Wilk test. The data were not normally distributed. Wilcoxon signed rank test was used to find the differences within the groups. And Kruskal Wallis test was used to find out the differences between the three groups. Results were considered significant at $\mathrm{p}<0.05$ and confidence interval of $95 \%$.

In this study, total of 90 subjects (age $=18$ - 25 years) were taken. All the subjects were randomly allocated in group A, B and C. Thus, all the groups had 30 subjects each. The subjects were within the age group of $18-25$ years with male $(n=20)$ and female $(n=70)$. Table 1 shows the demographic data and 2 shows the descriptive statistics of all subjects. Graph 1 shows the demographic distribution of gender in all groups.

\begin{tabular}{|l|c|}
\hline Groups & Age \\
\hline Self stretching $(\mathrm{n}=30)$ & $19.43 \pm 1.251$ \\
\hline Foam roller release $(\mathrm{n}=30)$ & $19.73 \pm 0.907$ \\
\hline Control $(\mathrm{n}=30)$ & $20.00 \pm 1.174$ \\
\hline
\end{tabular}

Table 1: Demographic data of all groups.

\begin{tabular}{|c|c|c|c|c|c|}
\hline & N & $\begin{array}{c}\text { Mini- } \\
\text { mum }\end{array}$ & $\begin{array}{c}\text { Maxi- } \\
\text { mum }\end{array}$ & Mean & $\begin{array}{c}\text { Standard } \\
\text { deviation }\end{array}$ \\
\hline Pre treatment & 90 & 10 & 15 & 11.49 & 1.651 \\
\hline Post treatment & 90 & 0 & 15 & 8.21 & 3.199 \\
\hline
\end{tabular}

Table 2: Descriptive statistics of all groups.

\begin{tabular}{|l|c|c|}
\hline Groups & $\begin{array}{c}\text { Mean rank (pre-post } \\
\text { treatment) }\end{array}$ & p value \\
\hline Self stretching & $15-0$ & $0.000^{* *}$ \\
\hline Foam roller release & $14-0$ & $0.000^{* *}$ \\
\hline Control & $0-0$ & 1.000 \\
\hline
\end{tabular}

Table 3: Mean rank of Wilcoxon Signed Rank test (pre-post treatment) outcome measure with p value.

\footnotetext{
**highly significant.
} 


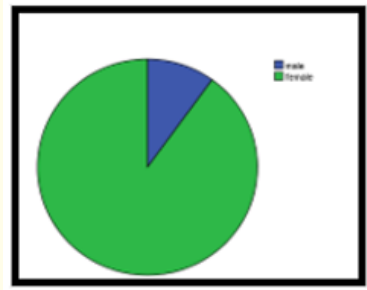

Self stretching

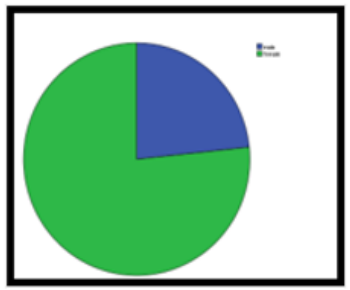

Foam roller release

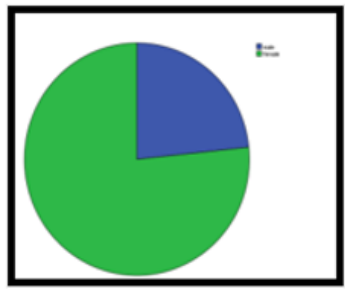

Control

Graph 1: Demographic distribution of gender in all groups (Blue: Male and Green: Female).

Table 3 shows the mean rank of Wilcoxon signed rank test of pre and post treatment outcome measures. The mean rank of pre and post treatment outcome measure of self stretching group was 15-0 with $\mathrm{p}$ value of 0.000 . The mean rank of pre and post treatment outcome measure of foam roller release group was 14-0 with $\mathrm{p}$ value of 0.000 . The mean rank of pre and post treatment outcome measure of control group was $0-0$ with $p$ value of 1.000 (Graph 2). Thus, there was statistical significant difference in pre and post treatment outcome measures within self stretching and foam roller release group but there was no statistical significant difference in the control group.

Table 4 shows the mean rank of post treatment outcome score of all the three groups using Kruskal Wallis test. When all the three groups were compared, there was significant difference between all the three groups.
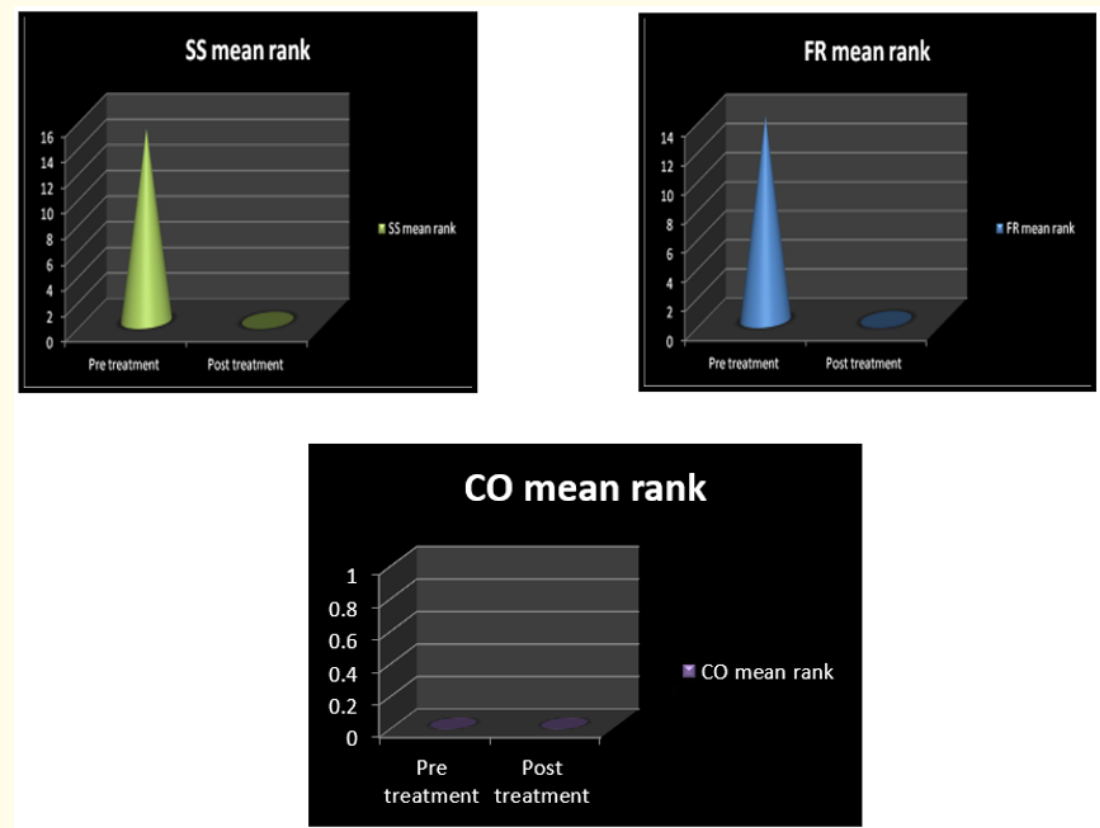

Graph 2: Pre and post mean rank of Ober test of self stretching, foam roller release and control group.

\begin{tabular}{|l|c|c|}
\hline Groups & Mean Rank & \multirow{2}{*}{ P value } \\
\hline Self Stretching & 36.33 & \multirow{2}{*}{$0.000^{* *}$} \\
\cline { 1 - 2 } Foam Roller Release & 26.50 & \\
\cline { 1 - 2 } Control & 73.67 & \\
\hline
\end{tabular}

\begin{tabular}{|l|c|}
\hline & P value \\
\hline Foam Roller Release - Self Stretching & 0.422 \\
\hline Foam Roller -Control & $0.000^{* *}$ \\
\hline Self Stretching- Control & $0.000^{* *}$ \\
\hline
\end{tabular}

Table 4: Mean Rank of all three groups using Kruskal Wallis test. ** Highly significant.
Table 5: Result of Post hoc analysis.

** Highly significant. 
Using Post hoc analysis, there was significant difference between foam roller-control group and self stretching-control group ( $p$ value 0.000 ). There was no significant difference between foam roller and self stretching group (P value 0.422 ).

\section{Discussion}

Tensor fascia lata is found to be tight in many individuals which can be the reason for various conditions. Flexibility of tensor fascia lata has been reported to improve the range of motion of hip joint and pelvis [25]. Therefore the purpose of this study was to find out the most effective technique for increasing the flexibility of tensor fascia lata and therefore we evaluated the immediate effect within and between self stretching, foam rolling and control group in subjects with TFL tightness. There was significant difference within self stretching $(p=0.001)$ and foam roller release group ( $p=0.001$ ) but there was no significant difference in control group ( $p>0.05$ ). When all the groups were compared, there was significant difference in all groups. The result of post hoc analysis stated increase in flexibility of tensor fascia lata was significant in all treatment groups with greater gains in self stretching and foam roller release group. There was no statistically significant difference between foam roller and self stretching group but when mean ranks were compared, foam roller release group was found to be more beneficial.

For the musculoskeletal system to function properly, not only all joints should maintain proper range of motion, but also the extensibility of the muscles, tendons, articular capsules and ligaments [28]. The possible mechanisms of significant change in self stretching within the group can be due to biomechanical changes that occur in muscle properties. There is some evidence from studies which suggest that if the muscle is held in a lengthened position for extended time period, it adapts by increasing the number of sarcomeres in series, sometimes referred to as myofibrillogenesis. It is theorized that sarcomere number addition occurs to maintain the greatest functional overlap of actin and myosin filaments in the muscle and may lead to relatively permanent form of muscle lengthening if the newly gained length is used on a regular basis in functional activities [27].

This study is in accordance with the study done by Bae., et al. [25] who demonstrated that the static stretching using a load can be actively utilized for low back pain in patient with shortened TFL which can improve the flexibility. The study supported that the application of stretching to the tensor fascia lata reduced the pain so that the tension of the lower extremity muscles was relieved and the imbalance in the functional aspect was improved to help recovery of daily life. The result of the study also showed that the application of stretching using a load on the tensor fascia lata connected from the pelvis to the lower extremity increased flexibility. In that study, the weight of the lower extremity of the subject was used for stretching without any tool. It can be easily used by patient with low back pain for flexibility.

The study done by Lempke., et al. [29] suggested that PNF stretching and static stretching both were effective intervention method for increasing hip flexion range of motion. The study was given for 30-second hold time and repeated for 3 to 4 times. The present study is in accordance with the duration of the stretching intervention. The study done by Kasunich., et al. [30] suggested the change in patient with low back and sacroiliac pain and proved that it is important to consider iliotibial band tightness as a possible cause of low back and sacroiliac pain and that proper management may need to include stretching of the iliotibial band along with trigger point therapy and chiropractic manipulation.

The possible mechanism in improving the flexibility of tensor fascia lata in foam roller release group to reduce soft tissue restriction is that self myofascial release technique involves small undulations back and forth over a dense foam roller, starting at the proximal portion of the muscle working down to the distal portion of the muscle or vice versa [24]. The small undulation place direct and sweeping pressure on the soft tissue of the body and foam roller. The friction generated from the undulations causes warming of fascia, promoting the fascia to take on a more fluid like form (known as the thixotropic property of the fascia), breaking up fibrous adhesions between the layers of fascia and restoring soft tissue extensibility [31]. There can also be neurophysiological mechanism which suggests that mechanical pressure from the foam roll influences tissue relaxation and pain reduction through central nervous system afferent input from the Golgi tendon reflex, mechanoreceptors (e.g. Golgi tendon organ), and nociceptors [32]. The present study is in accordance with the study done by Bushell., et al. [33] which suggested that consistent use of foam rolling produces increases in hip extension during dynamic lunge immediately preceding a dynamic activity.

The instructions on foam roller interventions can be in forms of video guided, live instruction and self guided. The study done by Cheatham., et al. [20] suggested that all the three forms of in- 
structions on foam roller interventions had similar improvements on knee range of motions and can be used interchangeably. The present study guided the subjects in form of live instruction. In the present study the duration of foam roller was given for 2 minutes which is in accordance with the study done by Cheatham., et al. [34] where foam rolling was given for 2 minutes with and without joint range of motion. The density of foam roller also plays an important role and the study done by Cheatham., et al. [35] stated that all three densities of foam roller soft, medium and hard had similar improvement in range of motion and selection of foam roller can be according to the patient's pain perception level. In the present study medium density foam roller was used. The control group showed no change in the tightness of tensor fascia lata which suggests the need of intervention.

In this study, when all the groups were compared simultaneously, there was no statistically significant difference between foam roller and self stretching group but when mean ranks were compared, foam roller release group was found to be more beneficial. The possible mechanism can be that static stretching has been associated with acute, undesirable reductions in performance in sporting movements, while self myofascial release has not, which can imply that self myofascial release can be used to gain similar improvement in range of motion and increasing flexibility of the muscles [2,17].

The intensity as well as duration of intervention of static stretching and foam roller release also plays an important role in the intervention. The subjects in the self stretching group were asked to stretch at intensity where they felt maximum stretch and stretching was given for 30 seconds for 3 repetitions whereas the study done by Bae., et al. [25] performed the stretching for 15 minutes per day for 2 weeks. According to the principles of athletic training, the optimal time for holding the stretched position indicates 30 seconds hold time repeated 3 to 4 times will provide the most beneficial results and this technique is considered much safer for sedentary or untrained individuals [36]. The subjects in foam roller release group performed the interventions using their body weight and duration of 2 minutes which was in accordance with the study done by Cheatham., et al. $[20,34]$ whereas different studies used different durations of intervention [2,17,18,23]. Due to difference in the methodology used by different studies can be one of the reasons for no statistical significant difference between foam roller release and static stretching group.
The study done by Mohr., et al. [18] and Skarabot., et al. [23] stated that use of foam roller along with static stretching due to difference in their mechanism and it can be that greater stimulus led to bigger improvement in joint ROM. The systematic review done by Cheatham., et al. [35] suggested that self myofascial release using a foam roller or roller massager have short term effects on increasing range of motion without negatively affecting muscle performance and may help attenuate decrements in muscle performance and DOMS after intense exercise.

\section{Limitations of Study}

- $\quad$ Long term follow up was not taken.

- $\quad$ Sample size was small.

- Study was done on healthy subjects with TFL tightness. Therefore, the study cannot be generalized to symptomatic population.

- Ideal cadence for the intervention was not followed.

- Study was limited to commercial foam roller with medium density.

\section{Conclusion}

There was statistical significant difference within self stretching and foam roller release group but when all three groups were compared simultaneously self stretching and foam roller release group were equally effective in reducing the tightness of tensor fascia lata.

\section{Future Recommendation}

- $\quad$ Study can be done with long term follow up.

- $\quad$ Study can be done taking large number of sample size.

- Study can be done on symptomatic population.

- Study can be done taking optimal type of foam roller for different populations.

- $\quad$ Study can be done taking proper cadence into account.

\section{Bibliography}

1. Barnes Mark F. "The basic science of myofascial release: morphologic change in connective tissue". Journal of Bodywork and Movement Therapies 1.4 (1997): 231-238.

2. MacDonald Graham Z., et al. "An acute bout of self-myofascial release increases range of motion without a subsequent decrease in muscle activation or force". The Journal of Strength and Conditioning Research 27.3 (2013): 812-821. 
3. Norkin Cynthia C and D Joyce White. "Measurement of joint motion: a guide to goniometry". FA Davis (2016).

4. Siler William L and Philip E Martin. "Changes in running pattern during a treadmill run to volitional exhaustion: fast versus slower runners". Journal of Applied Biomechanics 7.1 (1991): 12-28.

5. Barber F Alan and Allan N Sutker. "Iliotibial band syndrome". Sports Medicine 14.2 (1992): 144-148.

6. Kippers Vaughan and Anthony W Parker. "Toe-touch test: a measure of its validity". Physical Therapy 67.11 (1987): 16801684.

7. Ekman Evan F., et al. "Magnetic resonance imaging of iliotibial band syndrome". The American Journal of Sports Medicine 22.6 (1994): 851-854.

8. Orchard John W., et al. "Biomechanics of iliotibial band friction syndrome in runners". The American Journal of Sports Medicine 24.3 (1996): 375-379.

9. Peterson-Kendall, F., et al. "Muscles testing and function with posture and pain". US: Lippincott Williams and Wilkins Ltd (2005): 49-118.

10. Simmonds Nigel., et al. "A theoretical framework for the role of fascia in manual therapy". Journal of Bodywork and Movement Therapies 16.1 (2012): 83-93.

11. Fasen Jo M., et al. "A randomized controlled trial of hamstring stretching: comparison of four techniques". The Journal of Strength and Conditioning Research 23.2 (2009): 660-667.

12. Matsuo Shingo., et al. "Changes in force and stiffness after static stretching of eccentrically-damaged hamstrings". European Journal of Applied Physiology 115.5 (2015): 981-991.

13. Sefton JoEllen. "Myofascial release for athletic trainers, part I: Theory and session guidelines". International Journal of Athletic Therapy and Training 9.1 (2004): 48-49.

14. Couture Grace., et al. "The effect of foam rolling duration on hamstring range of motion". The Open Orthopedics Journal 9 (2015): 450 .

15. Le Gal Julien., et al. "Effects of self-myofascial release on shoulder function and perception in adolescent tennis players". Journal of Sport Rehabilitation 27.6 (2018): 530-535.
16. Grieve Rob., et al. "The immediate effect of bilateral self myofascial release on the plantar surface of the feet on hamstring and lumbar spine flexibility: a pilot randomized controlled trial". Journal of bodywork and Movement Therapies 19.3 (2015): 544-552.

17. Sullivan Kathleen M., et al. "Roller-massager application to the hamstrings increases sit-and-reach range of motion within five to ten seconds without performance impairments". International Journal of Sports Physical Therapy 8.3 (2013): 228.

18. Mohr Andrew R., et al. "Effect of foam rolling and static stretching on passive hip-flexion range of motion". Journal of Sport Rehabilitation 23.4 (2014): 296-299.

19. Murray Andrew M., et al. "Sixty seconds of foam rolling does not affect functional flexibility or change muscle temperature in adolescent athletes". International Journal of Sports Physical Therapy 11.5 (2016): 765.

20. Cheatham Scott W., et al. "Comparison of video-guided, live instructed, and self-guided foam roll interventions on knee joint range of motion and pressure pain threshold: a randomized controlled trial". International Journal of Sports Physical Therapy 12.2 (2017): 242.

21. Cheatham Scott W., et al. "Comparison of a vibration roller and a nonvibration roller intervention on knee range of motion and pressure pain threshold: a randomized controlled trial". Journal of Sport Rehabilitation 28.1 (2019): 39-45.

22. Su Hsuan., et al. "Acute effects of foam rolling, static stretching, and dynamic stretching during warm-ups on muscular flexibility and strength in young adults". Journal of Sport Rehabilitation 26.6 (2017): 469-477.

23. Škarabot Jakob., et al. "Comparing the effects of self-myofascial release with static stretching on ankle range-of-motion in adolescent athletes". International Journal of Sports Physical Therapy 10.2 (2015): 203.

24. Paolini John. "Review of myofascial release as an effective massage therapy technique". International Journal of Athletic Therapy and Training 14.5 (2009): 30-34.

25. Bae Hae-In., et al. "Effects of a static stretch using a load on low back pain patients with shortened tensor fascia lata". Journal of Exercise Rehabilitation 13.2 (2017): 227. 
26. Melchione William E and M Scott Sullivan. "Reliability of measurements obtained by use of an instrument designed to indirectly measure iliotibial band length". Journal of Orthopaedic and Sports Physical Therapy 18.3 (1993): 511-515.

27. Kisner Carolyn., et al. "Therapeutic exercise: foundations and techniques". Fa Davis (2017).

28. Shephard Roy J., et al. "On the generality of the "sit and reach" test: an analysis of flexibility data for an aging population". Research Quarterly for Exercise and Sport 61.4 (1990): 326-330.

29. Lempke Landon., et al. "The effectiveness of PNF versus static stretching on increasing hip-flexion range of motion". Journal of Sport Rehabilitation 27.3 (2018): 289-294.

30. Kasunich Norman J. "Changes in low back pain in a long distance runner after stretching the iliotibial band". Journal of Chiropractic Medicine 2.1 (2003): 37-40.

31. Sefton JoEllen. "Myofascial release for athletic trainers, part I: Theory and session guidelines". International Journal of Athletic Therapy and Training 9.1 (2004): 48-49.

32. Kelly, Shane and Chris Beardsley. "Specific and cross-over effects of foam rolling on ankle dorsiflexion range of motion". International Journal of Sports Physical Therapy 11.4 (2016): 544.

33. Bushell Jennifer E., et al. "Clinical relevance of foam rolling on hip extension angle in a functional lunge position". The Journal of Strength and Conditioning Research 29.9 (2015): 23972403.

34. Cheatham Scott W and Kyle R Stull. "Comparison of a foam rolling session with active joint motion and without joint motion: A randomized controlled trial". Journal of Bodywork and Movement Therapies 22.3 (2018): 707-712.

35. Cheatham Scott W and Kyle R Stull. "Comparison of three different density type foam rollers on knee range of motion and pressure pain threshold: a randomized controlled trial". International Journal of Sports Physical Therapy 13.3 (2018): 474.

36. Arnheim DD and Prentice WE. “Arnheim's Principles of Athletic Training: A Competency-based Approach". McGraw-Hill (2003).

\section{Assets from publication with us}

- Prompt Acknowledgement after receiving the article

- Thorough Double blinded peer review

- Rapid Publication

- Issue of Publication Certificate

- High visibility of your Published work

Website: https://www.actascientific.com/

Submit Article: https://www.actascientific.com/submission.php Email us: editor@actascientific.com

Contact us: +919182824667 